Terradillos Basoco, Juan Maria, Aporofobia y plutofilia: La deriva jánica de la política criminal contemporánea, J.M. Bosch Editor, Barcelona, 2020, 192 pp., ISBN 978-84-1220-153-6.

http://dx.doi.org/10.18543/ed-68(2)-2020pp411-419

\section{«Una obra actual, imprescindible y necesaria»}

Mientras estaba leyendo la obra del catedrático emérito doctor Juan María Terradillos Basoco, la verdad es que notaba mi conciencia cada vez más removida a medida que iban pasando las páginas. $\mathrm{Al}$ terminar de leer la obra estuve varias semanas pensando que lo que había escrito el profesor Terradillos merecía de alguna forma un humilde reconocimiento de quién le estaba leyendo, y por otra parte también que sirviese de alguna forma de altoparlante de tanto contenido que había podido disfrutar y aprender. Pero claro está que realizar una reseña a un insigne jurista como el Dr. Terradillos Basoco es una tarea que requiere mucha reflexión y máximo respeto ante todo por la figura del autor y lo que representa para los que alguna cosa sabemos y leemos sobre el Derecho penal y la Criminología.

Entre sentimientos encontrados de empezar a escribir o callar para siempre (algo que en mi fuero interno me decía que era imposible), decidí pedir consejo a una muy buena amiga y compañera de profesión, y fue ella la que me animó a que me lanzase a realizar esta reseña que el lector tiene ante sí.

Cuando uno lee por primera vez el título de Aporofobia y plutofilia: la deriva jánica de la política criminal contemporánea, la verdad es que resulta chocante, y a la vez produce ansia de saber cuál va a ser la temática real de la que tratará la obra.

El lector, cuando tiene en sus manos esta obra editada por el decano editor J.M Bosch, ya puede intuir algo con una portada velada, cuidada con el máximo detalle, que expresa de forma sencilla y sugerente el contraste duro y contundente entre la opulencia y la pobreza más estricta. Acudimos a la contraportada como cualquier lector que quiere saber qué es lo que va a leer, y se empieza a descubrir que se nos habla del Dios Jano, el de las dos caras, simbolizando la plutofilia y la aporofobia en relación a la aplicación de la ley.

El autor comienza con una cita de Negri de la que solamente indicaré el inicio, y que es el siguiente: «No lo olvidemos: estamos hablando del derecho penal y no de bromas...». 
Ya en la primera línea de la primera página de esta obra aparece una frase que se atribuye al escritor uruguayo Eduardo Galeano, que reza: «La justicia, como la serpiente, solo muerde a los descalzos». Y es aquí cuando ya empezamos a darnos cuenta de que la temática va a ser cuanto menos cruda y dura, pues también se nos cita la obra más conocida de Adela Cortina titulada Aporofobia, el rechazo al pobre, un desafio para la democracia (2017).

El profesor Terradillos comienza a situarnos ante un frenesí de situaciones contrapuestas entre los que van a pagar muy caro sus errores ante la justicia penal, como inmigrantes, prostitutas, vendedores del top manta, trabajadores en negro, raperos molestos, (supuestos) Yihadistas lobos solitarios, narcomenudeantes, los que hurtan de forma trivial y que además son rumanos, y trileros de mercadillo. Todo este grupo de personas frente a los que van a ser tratados de forma muy distinta por el Derecho penal, de forma mucho más benevolente como son: la scort de lujo, los falsificadores industriales al por mayor, los empresarios que contratan a trabajadores en negro o en el que hoy, de forma eufemística denominamos «dinero en $\mathrm{B}$ », defraudadores tributarios que se van a beneficiar de amnistías fiscales, influencers muy nocivos para la vida de otras personas, grandes narcotraficantes, organizaciones criminales rusas con inversiones inmobiliarias de más que dudosa procedencia que hemos tenido que sufrir durante años, autoridades corruptas que permiten malgastar el dinero de todos los contribuyentes. $\mathrm{Y}$ es que el profesor hace que nos acordemos de esas construcciones inútiles y ahora inservibles como podrían ser las llamadas «urbanizaciones fantasma» que jamás llegaron a terminarse por falta de liquidez con inversiones especulativas que perseguían una voracidad de lucro sin fin, y multitud de estructuras que se consideraban estructurales y fundamentales para nuestro país, como los aeropuertos, que se han quedado prácticamente sin sus principales protagonistas, que son los aviones y pasajeros, pasando también a convertirse en «aeropuertos fantasma» con un dispendio económico público estratosférico.

Ya en el primer capítulo el autor nos habla de los derechos económicos sociales y culturales que tendrían que ser pilares fundamentales de los estados avanzados democráticamente y que se ven damnificados por un cambio sustancial en la política criminal contemporánea que castiga de forma inmisericorde al más pobre, al que no puede aportar nada o casi nada a nuestra sociedad, y beneficia al más poderoso económicamente. Se contrapone el llamado «Derecho penal del enemigo» con el «Derecho penal del amigo».

De forma meridianamente clara, Terradillos nos indica que las estrategias político-criminales no respetan los principios fundamentales del Derecho penal, como son los de igualdad, lesividad, intervención mínima, proporcionalidad, reinserción, Derecho penal de hecho y presunción de inocencia; afirmaciones realmente inquietantes y que invitan a la reflexión, al debate y a la actuación para cambiar una situación como la descrita. 
El autor nos habla de economía desde 1913 hasta la globalización de nuestros días en la que la Lex mercatoria se impone ante la justicia, pues a los mercados solamente les importa la rentabilidad ante todo, en un contexto en el que las grandes corporaciones desregulan el Derecho penal y lo dejan en una intervención bajo mínimos que alcanza sólo a los más vulnerables.

Entrando ya en la corrupción, el autor nos habla de que va íntimamente unida a la criminalidad y a la política, indicando incluso con datos que la corrupción política en España lastra el crecimiento de la economía en un $1 \%$ del PIB anual. Todo ello conlleva una ineficiencia de asignación de recursos, una menor productividad, mayor desempleo, bajada de salarios, entre otros efectos que por mor de brevedad no relataremos. Y lo que es muy curioso es la afirmación que nos hace el profesor Terradillos cuando indica que la corrupción no es el principal problema que preocupa a los españoles, todo y con el daño socioeconómico que llega a producir, pues en situaciones graves de pandemia este factor pasó a ser una preocupación que ocupaba el cuarto lugar.

La desregulación por parte de los estados supuestamente avanzados en relación al Derecho penal es algo que también queda denunciado en esta obra, pues se pone de manifiesto la privatización o autorregulación de las conductas ilegales sobre todo en las grandes corporaciones. Y así pues, es preciso acercarnos al mundo del compliance penal, que se ha convertido en un auténtico negocio de certificaciones, algunas de ellas más que dudosas desde un punto de vista de rigurosidad jurídico-penal y criminológica, con «copiares y pegares» entre programas de cumplimiento, como ha denunciado la Fiscalía General del Estado en la Circular 1/2016, de 22 de enero, sobre la responsabilidad penal de las personas jurídicas conforme a la reforma del Código Penal efectuada por Ley Orgánica 1/2015.

En una situación en la que el capitalismo y la Lex mercatoria dominan, surgen una serie de colectivos muy vulnerables, como son los inmigrantes irregulares captados por mafias organizadas, y «contratados» para ser explotados laboral y/o sexualmente, que son ingresados en centros de internamiento (CIE) (de dudosa naturaleza jurídica), o bien expulsados por ser irregulares administrativamente, pues como personas que son, deberían de gozar del derecho a la libre circulación por el orbe, y con valentía lo denuncia Terradillos Basoco.

En pos de contextualizar, la obra del profesor de Terradillos estuvo fraguada en los tiempos más duros del estricto confinamiento, y nos hablaba de las carencias de la sanidad pública en España en los momentos más álgidos de la pandemia que estaba afectando a los colectivos más vulnerables, carencias sociales que estaban conculcando los derechos más elementales y fundamentales de nuestros ciudadanos.

La obra que aquí reseño se publicó en junio de 2020, y mientras estoy escribiendo estas líneas siendo ya el mes de diciembre de este año para el olvido, se corrobora de forma evidente lo que nuestro querido profesor y maestro, desgraciadamente vaticinaba. El 
día 10 de diciembre, en un barrio muy humilde de Badalona (El Gorg) fallecían al menos tres personas y quedaban 17 heridos que malvivían junto a entre 150 y 200 personas más, en situación de hacinamiento sin los más elementales servicios de luz y agua, la mayoría inmigrantes, los «sin papeles». ¿La causa?, según las autoridades, una vez más, el fuego de una vela, que ha acabado con la vida de estas personas de las que nos habla el profesor, de los que poco o nada tienen que aportar a nuestra sociedad, o ¿quizás sí? porque acaban de dejarse el TODO, su vida en tierra de España, un país que en teoría disfruta de un estado social y democrático de derecho y en el que tenemos que vivir situaciones como la que explico con estupor y vergüenza. Y no es la primera vez que esto sucede, pues en Reus, una anciana fallecía también en un incendio en pleno invierno de noviembre de 2016 porque se alumbraba con una humilde vela al no poder pagar las facturas de la luz. Y es que viene a mi recuerdo aquella frase que cantaba Rubén Blades y que dice: «si naces para martillo, del cielo te caen los clavos...» de aquel Pedro Navaja, donde se utilizaba para referirse al fatal y previsible destino de una persona

Más que nunca cobra pleno sentido el texto del profesor Terradillos que nos habla de forma pausada, razonada, directa y asertiva, de la pobreza y la desigualdad como marco económico de nuestro mundo neoliberal globalizado que excluye de forma deliberada, premeditada y alevosa a colectivos cada vez más vulnerables y desprotegidos como pueden ser los inmigrantes, los indigentes, los que caen en las listas inacabables del paro (hoy en ERTES), que ya parece ser algo asumido y característico de España.

El profesor nos ilustra con numerosos ejemplos como el del efecto Mateo de Merton, que afirma que «a quien mucho tiene, se le dará en abundancia; pero a quien poco, incluso lo poco que tiene se le quitará»».

$\mathrm{Y}$ es que, además, el impacto económico y social de la pandemia de la COVID-19 es resaltado específicamente en las letras de esta obra tan actual y necesaria. Obra necesaria para que no caigan en el olvido las necesidades de las personas sean de donde sean y tengan el color de la piel que tengan.

La desigualdad en la distribución de la riqueza y por lo tanto de las oportunidades de tener una vida digna son resaltadas en esta obra con nombres y apellidos de forma valiente y decidida. Se habla de ultra ricos, del fundador de Inditex que llegaba a acumular el 2,6\% del producto interior bruto de España en 2018 y que era de 1,2 billones de euros. También quedan reflejados los niveles de los sueldos de los altos ejecutivos del Ibex 35 que nada más y nada menos en 2018 percibían 23 veces el salario medio de sus empleados. Son unas cifras realmente escandalosas e insoportables. Con detalle se nos habla de informes diferentes que avalan que las diferencias sociales se acrecientan con el paso del tiempo.

Y la política criminal seguirá el camino de una política de exclusión social preexistente sustentada sobre unas bases de aporofobia y plutofilia, 
de castigo al más débil y trato preferente al cada vez más poderoso.

Citando a Jakobs se nos recuerda en esta obra que el expansionismo punitivista literalmente indica que «si la sociedad está inmersa en una tendencia hacia la disminución de los derechos de libertad, esta tendencia no será exclusivamente en el derecho penal» y que el Derecho penal está para proteger el statu quo de ciertas posiciones sociales, evidentemente las que gozan de una mejor situación socioeconómica. Y al hilo de lo dicho se indica que no se puede esperar que estas personas que gozan de una situación privilegiada traten a otras personas como tales siguiendo también a Jakobs.

Nuestro profesor nos habla del «gerencialismo» en el que se busca en primer lugar los rendimientos más que los resultados, y el ciudadano en esta situación dejará de ser titular de derechos para ser un simple consumidor de servicios, y las administraciones se dedicarán solamente a desarrollar programas políticos para aplicar valores neoliberales.

Es muy preocupante lo que nos cuenta el autor cuando explica que ya no estamos en una situación de garantizar derechos, sino de optimizar la oferta de servicios de control, y que las instituciones públicas ceden de forma paulatina ámbitos a la iniciativa privada, como puede ser el bien jurídico de la seguridad. Siendo la seguridad un bien jurídico de primerísimo orden que tiene que ser algo inherente de una sociedad avanzada, se nos recuerda que fundamentalmente en la situación actual, las fuerzas policiales se esfuerzan más en conseguir que la sociedad tenga una percepción determinada de las mismas, en lugar de dedicarse a concretar intervenciones efectivas. Y de esta forma se nos ilustra de forma directa en el sentido de que las actuaciones de la policía están más dedicadas a operaciones menores con un más fácil descubrimiento, descuidando a la vez zonas realmente peligrosas. No se ocupan los cuerpos policiales de la gran criminalidad, sino de aquello que es más visible, sencillo y podríamos decir de alguna forma «rentable» política y mediáticamente.

Cuando se nos habla del punitivismo se deja bien claro que la represión preventiva se dirige a la pequeña delincuencia, con la cual hay una tolerancia cero, incluso con detenciones discrecionales obedeciendo a estereotipos raciales o económicos, hecho que nos parece realmente grave.

Entrando ya en el apartado de la «deriva Jánica», se afirma que convergen el llamado funcionalismo, gerencialismo, y punitivismo. $\mathrm{Y}$ es que el Derecho penal de la aporofobia está inmerso en esta situación que jamás defiende al pobre, sino que incide de forma contundente en la represión del más débil, pues defender a esta tipología de seres humanos resulta incompatible con la generación de beneficios, algo inherente a la Lex mercatoria.

De forma clara el autor nos indica que las políticas criminales tienden a defender a ese delincuente que perpetra graves delitos que atacan frontalmente a los derechos económicos y sociales, siempre tras la protección de estructuras transnacionales muy bien preparadas que están bien protegidas 
por el compliance y autoridades corruptas, en una situación de confrontación clara entre el «Derecho penal del amigo» y el «Derecho penal de clase» o plutofilia.

Podemos leer en esta obra líneas que nos señalan que es preciso luchar contra aquellos criminales que defraudan a la Hacienda Pública y al sistema de la Seguridad Social, porque de lo contrario los derechos económicos y sociales serán algo ilusorio. Hay que acabar de una vez con la plutofilia. Se denuncia asimismo el gran desvío de beneficios empresariales hacia paraísos fiscales que merman a nuestra Hacienda pública, y por lo tanto a nuestro estado del bienestar, y choca realmente leer que estos paraísos fiscales no están en «repúblicas bananeras» sino en países de la Unión Europea que gestionan el $80 \%$ del dinero oculto. Es realmente preocupante cuando el profesor explica que estos paraísos tienen a sus «cooperadores necesarios» en empresas que cotizan en el Ibex 35, y además nos da nombres y apellidos de estos adláteres como son: el Banco de Santander, ACS, Repsol, entre otras corporaciones extremadamente poderosas.

Hay una crítica directa al Código Penal español en cuanto al abordaje de los delitos contra la Seguridad Social en beneficio de ese «Derecho penal del amigo» que favorece a quienes defraudan grandes cantidades al socaire de empresas económicamente potentes, en una situación que no supera en nuestro país a estándares ni de eficacia ni de democracia. Y de esta forma el principio de igualdad se ve atacado por el favor que reciben las clases poderosas inmersas en la plutofilia, protegidas por programas de cumplimiento en sus empresas que les servirán de auténtico parapeto frente a investigaciones judiciales, $y$ en detrimento de la auténtica administración de la justicia que debería de tratar a todos por igual, con un fiel.

Y como decíamos, por otra parte, tenemos la aporofobia, que es ese Derecho penal del indigente, el que está marginado socialmente, el que también está marginado desde el punto de vista de la administración de la justicia, pues la política criminal se ocupa de ello, para tratar al más pobre y desprotegido bajo el prisma muy cercano al del «Derecho penal del enemigo».

El profesor Terradillos critica las últimas reformas del Código Penal que son discriminatorias de aquellos que ya están discriminados por el sistema, y lo describe con minuciosidad, afirmando que las políticas criminales se dirigen a combatir la pobreza mediante la tipificación de comportamientos habituales de los que menos recursos disponen, en definitiva, se castigan los delitos de bagatela y no a la criminalidad a gran escala y con grandes organizaciones bien preparadas y organizadas para consumar el crimen con éxito.

Y nuevamente se nos recuerda a Galeano que afirma: «Los presos son pobres, como es natural, porque sólo los pobres van presos en países donde nadie va preso cuando se viene abajo un puente inaugurado, cuando se derrumba un banco vaciado o cuando se desploma un edificio construido sin cimientos».

Otra de las reflexiones del profesor que más me hizo reflexionar y que deben de hacer reflexionar a quienes 
tienen el poder de decidir políticamente y cambiar las cosas, es que en los tiempos del más duro confinamiento, cuando la sanidad española estaba colapsada, se concedieron homologaciones de títulos sanitarios de forma acelerada porque había una necesidad acuciante, y por otra parte, se aprobaron permisos de trabajo a jóvenes inmigrantes para que no se perdieran las cosechas, y itodo esto para qué?, pues justamente para que después de los peores momentos las autoridades no facilitasen documentación a toda esta mano de obra que había llegado como «trabajadores invitados».

Se critica también el llamado «efecto llamada» de la inmigración, pero se hace también inciso en la «fuerza centrífuga de la pobreza» controlada por las administraciones, y por las mafias y explotadores de mano de obra, de lo que se benefician los más poderosos. Y de esta forma se genera así todo un escenario xenófobo repleto de injusticia, de miseria e ignominia que conculcan los más elementales derechos humanos.

Hay una referencia expresa a las políticas de inclusión que han de acentuarse en los momentos más duros de la pandemia de la COVID-19, por las que se tuvieron que activar reglas fiscales comunitarias que dejaban en suspenso el pacto de estabilidad y crecimiento en todo el territorio de la Unión Europea, permitiendo a los estados el incumplimiento de objetivos de estabilidad presupuestaria y para fortalecer el sistema sanitario tan necesitado por la población. Se recuerda la necesariedad de una actuación del derecho positivo de garantía de las instituciones que no pueden caer en manos de las relaciones privadas solamente reguladas, o mejor dicho, autoreguladas, siempre guiadas por el afán de lucro.

Podemos leer también una referencia expresa a la necesaria protección de los derechos económicos, sociales y culturales como bienes jurídico penales dignos de ser protegidos a través de distintos instrumentos jurídicos internacionales que, en la realidad, las más de las veces quedan entendemos en declaraciones de intención más que en una efectiva concreción.

Es de sumo interés la reflexión que indica que el estado no puede derivar sus funciones a instancias privadas, a las empresas, siendo solamente un supervisor, pues ha de ir a la raíz de los problemas para poder conseguir una verdadera igualdad en los derechos económicos sociales y culturales del hombre ciudadano.

Se dedica también en esta obra un epígrafe a los derechos económicos, sociales y culturales y a la corrupción política, que en definitiva será la que dañe a los primeros de forma contundente y con la connivencia entendemos de las administraciones y de los que realmente ostentan el poder socioeconómico. La política criminal de un país cuando lo que busca es la justicia social, ha de actuar sobre los pilares que sustentan el modelo económico que apoya a la corrupción que en este país parece ser ya endémica e inveterada.

Podemos apreciar también una crítica al sistema procesal penal, que de todos es sabido que es tedioso, y que está en la mayoría de ocasiones colapsado, precisándose una auténtica 
reforma profunda, meditada y estudiada con sosiego. Se señala a la Fiscalía como elemento imprescindible para afrontar a la corrupción, pero entendemos que ha de ser una Fiscalía que no tenga dependencia del Ejecutivo, pues entonces la imparcialidad queda en entredicho, y por ende también la intervención policial de las correspondientes investigaciones de los delitos.

Sobre la globalización se nos explica que también viene ligada a la delincuencia internacional, que requerirá respuestas globales y de una justicia con carácter universal sin barreras a la persecución del delito cada vez perpetrado con más complejidad, pues la libre circulación de mercancías, personas y capitales favorece al delincuente con la deslocalización de actividades y dispersión de beneficios. Se requiere una legislación penal en armonía, con carácter internacional que pueda combatir contra esta tipología de crímenes transnacionales.

Sobre la protección del derecho al trabajo se recuerda de forma muy acertada que ha de haber una intervención penal cuando hay ataques graves a ese derecho tan importante para el hombre, que en definitiva creemos que es ganarse la vida dignamente, $y$ que es intolerable que hoy día en pleno siglo XXI siga habiendo discriminación y xenofobia en este ámbito tan sensible e importante para una sociedad avanzada, o al menos que pretenda serlo.

Íntimamente ligado al derecho al trabajo está el derecho a la manifestación y al derecho de huelga que el profesor denuncia que es atacado directamente comportando ello en la mayor parte de las ocasiones sanciones administrativas a los sujetos activos, mientras que los trabajadores es habitual que paguen con su libertad mediante el ingreso en prisión provisional, hecho que nos parece sumamente revelador y muy grave.

De forma abierta se denuncia el trabajo forzoso y la esclavitud que también existen en este siglo XXI, cuando ello ya debería de ser algo que hubiese pasado a la historia. Y se denuncia otro de los graves problemas existentes en el mercado laboral, que es la discriminación por ejemplo, por razón de sexo, que sigue existiendo, y que no tiene el castigo disuasorio suficiente.

De forma muy acertada se hace referencia a los riesgos medioambientales y al desarrollo sostenible que de una vez por todas requiere políticas decididas de abordaje para no llegar a un punto de retorno imposible. Y el profesor señala que hay un auténtico abuso creciente de los recursos naturales debido a esa finalidad del marco capitalista que es la obtención del máximo beneficio económico sin importar cueste lo que cueste y se dañe a quien se dañe. Y ello sin que exista una protección penal decidida y eficaz para los que atentan contra el bien jurídico tan preciado del medio ambiente, que en definitiva es nuestro hábitat, y lo que vamos a dejar a nuestras generaciones futuras. Esta cuestión es de máxima actualidad, pues el Secretario General de la ONU, António Guterres, ha dicho hoy, 15 de diciembre de 2020: «Estamos en guerra con la naturaleza y hay que hacer las paces»».

Entrando en el ámbito tributario, se denuncia que la protección penal existente favorece a los poderosos, presentándose excepciones a los 
principios generales fundamentadores del Código Penal, sin que exista un motivo claro de política criminal, más que el del criterio «Del derecho penal del amigo» que se ejemplifica también en esta obra. Y así también sucede y denuncia el profesor con los fraudes a la Seguridad Social que solamente van a comportar castigo a los más débiles, quizás a aquellos que defraudan para conseguir algunos pocos euros para llegar a fin de mes, y no a las grandes mafias que disponen de mano de obra barata e incluso ilegal o cuanto menos inmoral en cuanto a sueldos y condiciones laborales.

Hemos querido de alguna forma subtitular esta obra como una obra actual, imprescindible y necesaria.

ACTUAL, porque está planteando una serie de problemáticas sociales, políticas, culturales, medioambientales, criminales, ideológicas, etc. que son patentes y que el profesor Terradillos Basoco ejemplifica con máximo detalle, con nombres y apellidos, con coraje, sin ambages.

Es una obra IMPRESCINDIBLE, porque entendemos que las generaciones actuales y las que vendrán deberían tener a este trabajo como lectura de cabecera para comprender lo que estamos viviendo y lo que hemos de vivir, y por supuesto; se trata de una obra NECESARIA para que de una vez por todas lo que aquí se denuncia con todo lujo de detalles, sin alarmismo, con argumentos y de forma completa y compleja, provoque a los agentes sociales que pueden y deben realizar cambios, que los hagan, pero que los hagan ya, sin perder más el tiempo en diatribas y crispaciones en los estrados de la cámara baja por poner un ejemplo, o en los foros de los medios de comunicación cada vez más mediatizados. Se ha terminado el tiempo de la queja, de la paciencia, de la pasividad del «españolito» sufrido que lo aguanta todo consolándose con el inveterado comentario en la cola del pan (si es que le llega para poder comprarlo), pues lo que se requiere de una vez por todas es que se termine con esta doble cara del Dios Jano proyectada en nuestra sociedad y nuestro ordenamiento jurídico penal, que se termine con la antítesis indecente, inmoral e indignante de la aporofobia y la plutofilia que describe de forma magistral nuestro querido maestro y admirado catedrático Emérito de Derecho Penal, el Dr. Juan María Terradillos Basoco, a quien solamente me queda una palabra que decirle: gracias.

En Reus a, 15 de diciembre de 2020

Alfredo Abadías Selma

Profesor Contratado Doctor de Derecho Penal y Criminología Universidad Internacional de La Rioja UNIR 


\title{
TerRadillos Basoco, Juan Maria, Aporofobia y plutofilia: La de- riva jánica de la política criminal contemporánea, J.M. Bosch Editor, Barcelona, 2020, 192 pp., ISBN 978-84-1220-153-6.
}

\author{
http://dx.doi.org/10.18543/ed-68(2)-2020pp411-419
}

\section{Copyright}

Estudios de Deusto es una revista de acceso abierto, lo que significa que es de libre acceso en su integridad. Se permite su lectura, la búsqueda, descarga, distribución y reutilización legal en cualquier tipo de soporte sólo para fines no comerciales, sin la previa autorización del editor o el autor, siempre que la obra original sea debidamente citada y cualquier cambio en el original esté claramente indicado

Estudios de Deusto is an Open Access journal which means that it is free for full access, reading, search, download, distribution, and lawful reuse in any medium only for non-commercial purposes, without prior permission from the Publisher or the author; provided the original work is properly cited and any changes to the original are clearly indicated. 